\title{
Solid State NMR Analysis of Fluorinated Single-Walled Carbon Nanotubes: Assessing The Extent of Fluorination
}

\author{
Lawrence B. Alemany, Lei Zhang, Liling Zeng, Christopher L. Edwards, \\ and Andrew R. Barron*
}

Richard E. Smalley Institute for Nanoscale Science and Technology, Department of Chemistry, and Department of Mechanical Engineering and Materials Science, Rice University, Houston, Texas 77005, USA

Supplementary Materials 
Table S1. Calculated and experimental C1s binding energies for carbons in pure SWNTs, graphites, F-SWNTs and model compounds. ${ }^{a}$

\begin{tabular}{|c|c|c|}
\hline $\begin{array}{l}\text { Types of carbons } \\
\text { and examples }\end{array}$ & $\begin{array}{l}\text { Estimated } \\
(\mathrm{eV}) \\
\end{array}$ & $\begin{array}{l}\text { Experimental } \\
(\mathrm{eV})\end{array}$ \\
\hline $\mathrm{sp}^{2}$ carbons & & 284.3-284.8 b,c,d,e,f \\
\hline C-C-F & $285.0-285.7$ & $285.8-286.4 \mathrm{~b}, \mathrm{~h}$ \\
\hline$\underline{\mathrm{C}}-\mathrm{O}$ & $285.7-286.3$ & $285.8-286.7 \mathrm{c}, \mathrm{d}$ \\
\hline $\mathrm{CH}_{3} \mathrm{CH}_{2} \mathrm{OH}$ & & $286.3 \mathrm{e}$ \\
\hline $\mathrm{CH}_{3} \mathrm{CO}(\mathrm{O}) \mathrm{CH}_{2} \mathrm{CH}_{3}$ & & 286.9 e \\
\hline$\underline{\mathrm{C}}-\mathrm{F}$ & $287.0-288.0 \mathrm{i}$ & 287.1-290.8 b,c,d,f,g \\
\hline$\underline{\mathrm{C}}(\mathrm{O}) \mathrm{F}$ & & $289.3 \mathrm{~d}$ \\
\hline carbonyl & & $287.6 \mathrm{c}$ \\
\hline $\mathrm{CH}_{3} \mathrm{C}(\mathrm{O}) \mathrm{CH}_{3}$ & & $287.9 \mathrm{e}$ \\
\hline carboxyl & & $288.3-288.5 \mathrm{c}, \mathrm{d}, \mathrm{f}$ \\
\hline $\mathrm{CH}_{3} \mathrm{C}(\mathrm{O}) \mathrm{OH}$ & & $289.3 \mathrm{e}$ \\
\hline $\mathrm{CF}_{2}$ & 289.3-290.8 & 290.3-291.4 hf \\
\hline $\mathrm{CF}_{3}$ & 291.8-294.4 & 292.5-293.0 h,f \\
\hline $\mathrm{CO}_{2}$ & & $291.9 \mathrm{e}$ \\
\hline
\end{tabular}

a Primary chemical shift caused by fluorine: $2.5-3.2 \mathrm{eV}$, average shift: $2.9 \mathrm{eV}$. Secondary shift caused by fluorine: $0.4-0.9 \mathrm{eV}$, average shift: $0.7 \mathrm{eV}$. Primary shift by oxygen is about $1.5 \mathrm{eV}$. Normally we don't consider the secondaryshift by oxygen. (a) Briggs, D., Seah, M. P., Practical Surface Analysis by Auger and X-ray Photoelectron Spectroscopy. John Wiley \& Sons Ltd. 1983. (b) Cox, D. M.; Cameron, S. D.; Tuinman, A.; Gakh, A.; Adcock, J. L.; Compton, R. N.; Hagaman, 
E. W.; Kniaz, K.; Fischer, J. E.; Strongin, R. M.; Cichy, M. A.; Smith, A. B., J. Am. Chem. Soc. 1994, 116, 1115-1120.

b Wang, Y-Q.; Sherwood, P. M. A., Chem. Mater. 2004, 16, 5427-5436.

c Marcoux, P. R.; Schreiber, J.; Batail, P.; Lefrant, S.; Renouard, J.; Jacob, G.; Albertini, D.; Mevellec, J-V., Phys. Chem. Chem. Phys., 2002, 4, 2278.

d Shofner M. L.; Khabashesku, V. N.; Barrera, E. V., Chem. Mater, 2006, 18, 906.

e Chastain, J.; King Jr., R. C.; Handbook of X-ray-Photoelectron Spectroscopy. Physical Electronics, Inc., 1995.

f Lee, Y. S.; Cho, T. H.; Lee, B. K.; Rho, J. S.; An, K. H.; Lee, Y. H. J. Fluorine Chem. 2003, 120, 99.

g Plank N. O. V.; Forrest G. A.; Cheung, R.; Alexander A. J. J. Phys. Chem. B. 2005, 109, 22096.

h Pehrsson, P. E.; Zhao, W.; Baldwin, J. W.; Song, C.; Liu, J.; Kooi, S.; Zheng, B. J. Phys. Chem. B 2003, 107, 5690.

i This value is only for carbons directly attached to one fluorine and at least two atoms away from other fluorines. For carbons directly attched to one fluorine and one atom away from other fluorines, the calculated binding energy can be from 287.4 to $290.7 \mathrm{eV}$. 


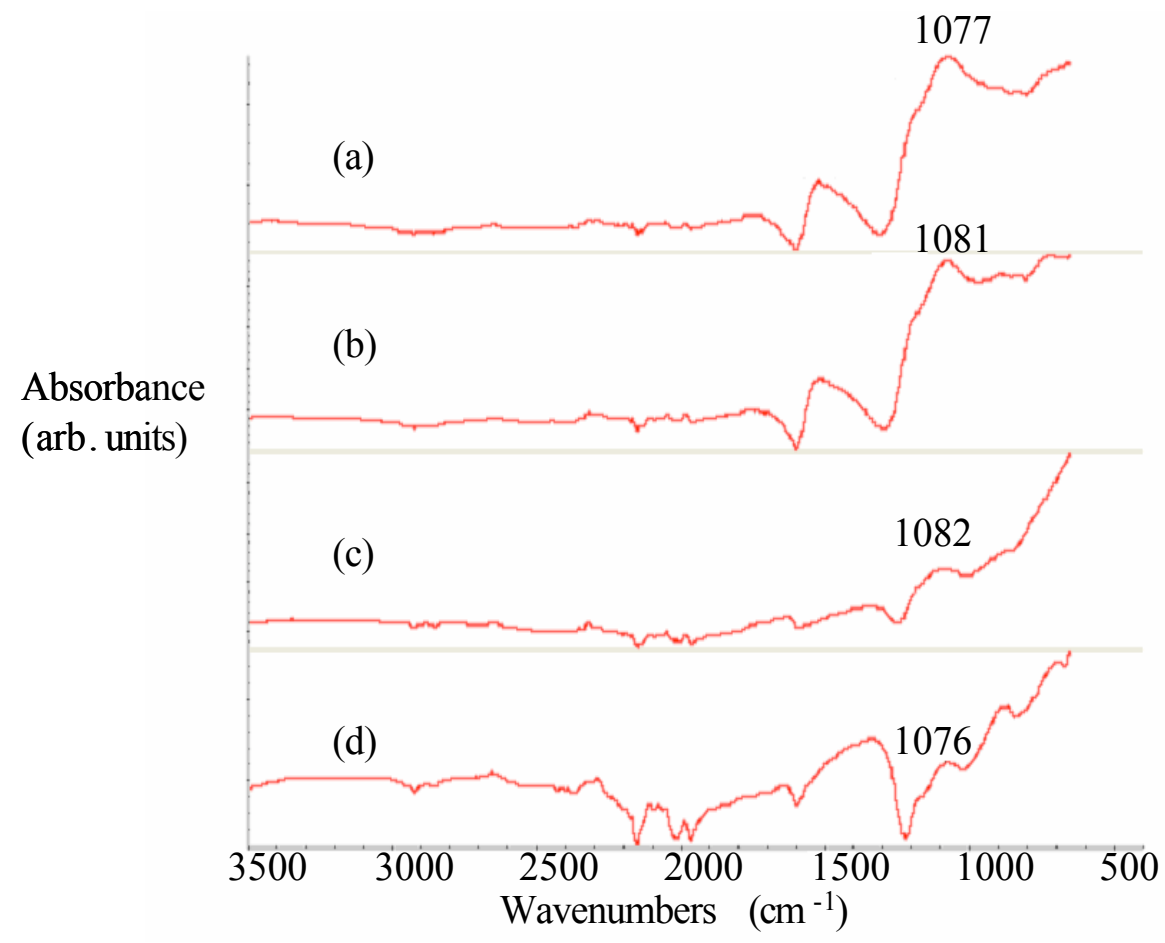

Figure S1. ATR-FTIR spectra of F-SWNTs (a) after heating to (b) 400, (c) 450, and (d) $550^{\circ} \mathrm{C}$. 